\title{
Late recurrent hemarthrosis following elective total knee arthroplasty
}

\author{
Matthew J Grosso ${ }^{*}$, Sophie Chheang ${ }^{2}$ and William Macaulay ${ }^{3}$
}

\begin{abstract}
${ }^{*}$ Correspondence
Matthew J Grosso

Center for Hip and Knee Replacement Columbia University Medical Center 622 West 168th Street, PH 11 New York, NY Email: mjg2235@cumc.columbia.edu

${ }^{1}$ Center for Hip and Knee Replacement Columbia University Medical Center 622 West 168th Street, PH 11 New York, NY 10032

IInterventional Radiology New York Presbyterian Hospital/Columbia University Medical Center 622 West 168th Street New York, NY 10032 ${ }_{3} \mathrm{NYU}$ Langone Orthopedic Hospital Department of Orthopaedic Surgery 46 E 20th St. 1st Floor New York, New York 10003
\end{abstract}

Received: May 8, 2018

Accepted: Jun 16, 2018

Published: Jun 20, 2018

\begin{abstract}
In certain scenarios following total knee arthroplasty (TKA), vascular complications can present as recurrent hemarthrosis, resulting in continued pain and restricted range of motion post-operatively. We present a 77 year old female who underwent a right TKA. She had an uncomplicated recovery for 1 year following the TKA, but then developed episodic knee pain, with pain and swelling, which resulted in limitations to ambulation. Work-up was negative for infection or hardware related complications. At presentation to our office 2 years following the right TKA, aspiration showed thick hemarthrosis from the right knee. Right lower extremity angiogram demonstrated a nontumoral blush indicative of chronic synovial hyperemia. Patient underwent successful transarterial coil and selective embolization of the responsible geniculate arteries, resulting in complete resolution of symptoms at 2 years follow-up. Physicians and surgeons should be aware of vascular complications following TKA that can occur at mid or long term follow-up which may require therapeutic embolization for resolution of symptoms.
\end{abstract}

Keywords: Hemarthrosis, Total knee arthroplasty, Embolization, Geniculate arteries.

\section{Introduction}

Vascular complications following total knee arthroplasty (TKA) are rare. In certain scenarios, vascular complications can present as recurrent hemarthrosis, resulting in continued pain and restricted range of motion post-operatively. Rapid and appropriate identification and treatment are critical to ensuring and minimizing risks for implant failure. There are a few case reports describing recurrent hemarthrosis, most commonly occurring in the acute setting ( $<3$ months postoperatively), that responded well to therapeutic embolization [1-4]. There are a number of reported etiologies of recurrent hemarthrosis following TKA, with the most prevalent being the development of traumatic pseudoaneurysms, and synovial hyperemia [5-8]. Hemophilia is a reported risk factor for developing this condition. We present a case of late ( $>1$ year) recurrent hemarthrosis following elective TKA.

\section{Case History}

We present a 77 year old female with a history of hypothyroidism and bilateral knee and hip replacements. Her most recent procedure was a right TKA, which occurred in April, 2013. The procedure and initial recovery were uncomplicated. She had an uneventful recovery for 1 year following the TKA, with a complete return to her activities of daily living. At 12 months post-operatively she developed episodic knee pain, with pain and swelling, which resulted in limitations to ambulation. The episodes lasted 2-3 days. She had approximately 12 of these episodes. Prior work-up prior to presentation to our office was negative for hardware or infection related complications. She presented to our office 2 years following the right TKA (June, 2015). She denied episodes of fevers or chills, or any history of trauma to the knee. Her medications included aspirin 81 daily, levothyroxine, 
lisinopril and pregabalin. An aspiration of the right knee was performed. On examination, her right knee demonstrated a moderate effusion. There was no visible erythema. She had a limited active range of motion 0-80 degrees, with significant pain. Patient was unable to ambulate at time of presentation secondary to pain. Aspiration demonstrated a thick hemarthrosis. Cultures from the aspirate were negative for an infectious process. Radiographs demonstrated a total knee replacement in good alignment with no evidence of loosening. She was admitted as an inpatient, and interventional radiology consulted for potential vascular intervention.

A right lower extremity angiogram was performed. The angiogram demonstrated no evidence of significant atherosclerotic disease or pseudoaneurysm of the right femoral and popliteal arteries. However, it showed evidence of nontumoral blush indicative of chronic synovial hyperemia (Figure 1a). The patient underwent successful transarterial coil and selective embolization of the lateral superior geniculate artery and the medial superior geniculate artery (Figure 1b).
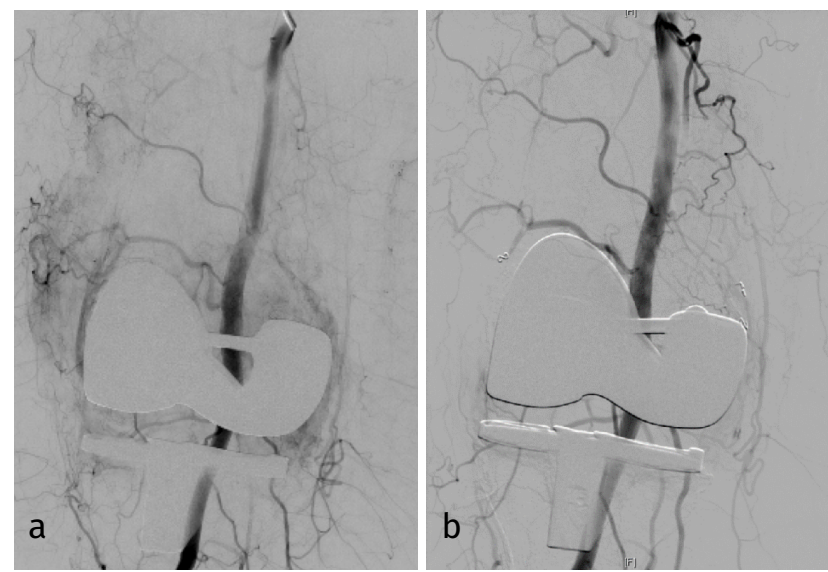

Figure 1: (1a) Right lower extremity angiogram demonstrated nontumoral blush consistent with chronic synovial hyperemia. (1b) Transarterial coil and selective embolization of the lateral superior geniculate artery and the medial superior geniculate resulted in resolution of the non-tumoral blush.

Following embolization, there was complete resolution of the non-tumoral brush, as well as complete symptomatic resolution with no recurrent episodes of hemarthroses at 2 years follow-up. Her follow-up exam demonstrated active range of motion from 0-120 degrees, no erythema or induration, and a non-antalgic gait.

\section{Discussion}

Recurrent spontaneous hemarthrosis following TKA is uncommon, occurring in less than $1 \%$ of cases [9]. However, when it does occur, this condition can cause debilitating symptoms, significantly impacting quality of life following TKA. Diagnosis can be difficult, especially if the providers are not familiar with this complication. This case reports of late presenting recurrent hemarthrosis secondary to synovial hyperemia highlights the importance of awareness of vascular complications following TKA which may require therapeutic embolization.

The first step in successful treatment of recurrent hemarthrosis is appropriate diagnosis. Initial diagnostic work-up should rule out more common causes of recurrent effusions and pain following TKA, such aseptic loosening, or infectious etiologies. Once these complications are eliminated, further work-up can be initiated. Needle aspiration should be performed in the early stages. An office aspiration can be both therapeutic, as well as diagnostic. The aspiration is needed to definitively rule out infection. In addition, the presence of frank blood can alert the surgeon to other potential causes of unexplained hemarthrosis. Initial treatment for atraumatic and aseptic hemarthrosis should be conservative management, consisting of cool, elevation and rest [10,11]. In a small series by Kindsfater and Scott, they reported resolution of symptoms in $9 / 30$ patients following conservative management. However, if conservative management is not successful, then lower extremity angiography should be performed. In our case report, the patient presented after 12 recurrent episodes, and had already failed conservative measures.

The most common causes for recurrent hemarthrosis in the literature are genicular pseudoaneurysm formation and synovial hypertrophy/hyperemia [3,5-7,10]. Genicular pseudoaneurysm formation typically occurs in the acute setting, while synovial hypertrophia often has a delayed presentation. In available case reports and case series, the mean time between arthroplasty and first instance of hemarthrosis was 22-26 months. Both etiologies often require non- 
orthopedic interventions, and we recommend consulting vascular surgery or interventional radiology. Historically, synovial hyperemia was treated with more invasive surgical intervention, which included either arthroscopic or open synovectomy. In the small case series available, recurrence rates were low with these interventions $[11,12]$. More recent cases reports have focused on the less invasive intervention of arterial embolization [3]. Weidner et al. reported resolution of hemarthrosis in 12 of 13 patients following geniculate arterial embolization. We reported similar success, with no recurrence of symptoms at 2 years follow-up.

In summary, physicians and surgeons should be aware of vascular complications following total knee arthroplasty which may require therapeutic embolization for resolution of symptoms. As demonstrated in this case report, these complications can occur at mid or long term followup. Initial management should include aspiration, to rule out infection as a cause of acute swelling and pain in a previously "forgotten joint". If frank blood is found (hemarthrosis) and fracture is ruled out, femoral arteriography should be considered, with selective embolization as needed.

\section{Copyrights}

(c) Matthew J Grosso et al. 2018; licensee OA Journal of Radiology. This is an Open Access article distributed under the terms of the Creative Commons Attribution License (http:// creativecommons.org/licenses/by/4.0), which permits unrestricted use, distribution, and reproduction in any medium, provided the original work is properly credited.

\section{References}

1. Karataglis D, Marlow D, Learmonth DJA (2006) Atraumatic haemarthrosis following total knee replacement treated with selective embolisation. Acta Orthop Belg. 72: 375-377.

2. Rukavina A, Kerkhoffs GMMJ, Schneider P, et al. (2010) Recurrent hemarthrosis after total knee arthroplasty. Knee Surg Sports Traumatol Arthrosc. 18: 898-900.

3. Weidner ZD, Hamilton WG, Smirniotopoulos J, et al. (2015) Recurrent Hemarthrosis Following Knee Arthroplasty Treated with Arterial Embolization. J Arthroplasty. 30: 2004-2007.

4. Pritsch T, Pritsch M, Halperin N (2003) Therapeutic embolization for late hemarthrosis after total knee arthroplasty. A case report. J Bone Joint Surg Am. 85-A: 18021804

5. Ibrahim M, Booth RE, Clark TWI (2004) Embolization of traumatic pseudoaneurysms after total knee arthroplasty. J Arthroplasty. 19: 123-128.

6. Rodriguez-Merchan EC, Jimenez-Yuste V, Gomez-Cardero P, et al. (2014) Severe postoperative haemarthrosis following a total knee replacement in a haemophiliac patient caused by a pseudoaneurysm: early treatment with arterial embolization. Haemophilia. 20: e86-89.

7. Gajjar SM, Platts A, Dowd G (2010) Synovial hypertrophy causing recurrent hemarthrosis after total knee replacement. J Knee Surg. 23: 25-28.

8. van Baardewijk LJ, Hoogeveen YL, van der Geest ICM, et al. (2018) Embolization of the Geniculate Arteries Is an Effective Treatment of Recurrent Hemarthrosis Following Total Knee Arthroplasty That Can Be Safely Repeated. J Arthroplasty. 33: 1177-1180.

9. Langkamer VG (2001) Local vascular complications after knee replacement: a review with illustrative case reports. Knee. 8 : 259-264.

10. Mounasamy V, Dawson C, Cui Q, et al. (2007) Popliteal artery pseudoaneurysm following total knee arthroplasty: a case report. Eur J Orthop Surg Traumatol. 17: 313-315.

11. Kindsfater K, Scott R. (1995) Recurrent hemarthrosis after total knee arthroplasty. J Arthroplasty. 10: S52-S55.

12. Ohdera T, Tokunaga M, Hiroshima S, et al. (2004) Recurrent hemarthrosis after knee joint arthroplasty: etiology and treatment. J Arthroplasty. 19: 157-161. 